\title{
Molluscan Fauna from Core 25B, Whipray Basin, Central Florida Bay, Everglades National Park
}

\author{
By
}

Carleigh A. Trappe and G. Lynn Brewster-Wingard

U.S. Geological Survey, Reston VA

\section{Open-File Report 01-143}

This report is preliminary and has not been reviewed for conformity with U.S. Geological Survey editorial standards or with the North American Stratigraphic Code. Any use of trade, product, or firm names is for descriptive purposes only and does not imply endorsement by the U.S. Government. 


\section{TABLE OF CONTENTS}

$\begin{array}{ll}\text { Abstract } & 1\end{array}$

Introduction $\quad 1$

Setting 2

Acknowledgements 4

Methods $\quad 5$

Core Collection $\quad 5$

Processing and Analysis $\quad 5$

Age Model $\quad 5$

$\begin{array}{ll}\text { Results } & 10\end{array}$

$\begin{array}{ll}\text { Discussion } & 12\end{array}$

$\begin{array}{ll}\text { Conclusions } & 16\end{array}$

$\begin{array}{lc}\text { References } & 18\end{array}$

\section{FIGURES}

1) Map showing the location of Whipray Basin and the cores

$5 \mathrm{G}$ and 25B within Florida Bay

2) Q-mode cluster diagram $\quad 8$

3) Percent abundance of select species in core, and total abundance and faunal richness for each sample

4) Plot of sub-aquatic vegetation trends within core 25B

\section{TABLES}

1) Molluscan occurrence table, recorded as percent abundance in each sample

2) Age-depth relationship for Core $25 \mathrm{~B}$ based on sedimentation rates calculated from ${ }^{210} \mathrm{~Pb}$ 


\title{
Molluscan Fauna from Core 25B, Whipray Basin, Central Florida Bay, Everglades National Park
}

\begin{abstract}
Molluscan assemblages preserved in an 80-cm core from Whipray Basin in central Florida Bay, Everglades National Park, illustrate changes in the environmental conditions within the basin over the last two centuries. Salinity remained polyhaline to euhaline throughout the time of deposition (1800-1997), with alternating periods of stability and increased fluctuations. Since 1800, a Brachidontes assemblage has characterized Whipray Basin and the dominant faunal components have remained the same in terms of presence and absence of species. However, patterns of dominance and diversity within the Brachidontes assemblage have changed and these changes indicate fluctuations in the environment.

The period from 1815 to 1857 was distinguished by an abundance of molluscs dwelling on seagrass and sub-aquatic vegetation. Faunal richness and abundance were high and stable, and epiphytic molluscs flourished. Polyhaline conditions existed, although periods of slightly lower salinities occurred. The period from 1862 to 1894 appears unstable based on fluctuations in molluscan faunal richness, abundance, and dominant species. The epiphytic molluscs experienced significant shifts $( \pm>30 \%)$ associated with changes in sub-aquatic vegetation. The changes in epiphytic molluscs from 1871 to 1913 may be indicative of a seagrass die-off. The period from 1899 to 1950 was the most stable section of the core in terms of changes in the molluscan fauna. Faunal richness and abundance reached highs of 31 groups and 726 individuals per sample during this period and epiphytic molluscs were prevalent. Beginning in 1955, faunal groups experienced high amplitude fluctuations in abundance; this pattern continued through the second half of the $20^{\text {th }}$ century. Fluctuating salinity, changes in vegetation, and reduced water quality (low $\mathrm{O}_{2}$, increased nutrients and/or reduced clarity) oxygen supply) have characterized the past 50 years. These changes preceded a seagrass die-off in 1987-88 and may be related to the causes of the die-off. Whether the cause of the changes seen in Whipray Basin is natural or a combination of natural and anthropogenic factors, the amount of change in the molluscan fauna in the last 50 years clearly exceeds the preceding 150 years.
\end{abstract}

\section{INTRODUCTION}

Worldwide, coastal environments are gaining more attention as significant changes are becoming evident. Every coastal environment is subject to different natural and anthropogenic factors and before effective restoration can proceed it is necessary to identify the significant forces behind the changes. Storms, changes in sea level, and variation in rainfall and climate can greatly alter an environment. While the effects of such changes may be dramatic, they are natural trends and therefore are part of the evolution of the system. Anthropogenic forces, however, can be managed to prevent 
further unnatural changes to an ecosystem. Humans have increasingly impacted coastal environments by changing the flow of water, and increasing pollution and nutrient runoff through development.

South Florida has recently been the focus of national attention following the passage of the "Everglades Forever Act" in 1994, which mandates the return of the Everglades to its "natural state." Everglades National Park encompasses Florida Bay, which is the downstream recipient of terrestrial runoff from much of southern Florida. The goal of the USGS's "Ecosystem History of Florida Bay and the Southwest Coast" project is to determine the nature and timing of changes to the ecosystem of south Florida and what agents are responsible for those changes. In developing a model for management and restoration of the ecosystem, it is essential to separate human-induced changes from natural changes. Analysis of past changes provides a means to predict the response of the system to future change.

Changes in the environment, whether natural or anthropogenic, ultimately cause a change in the biological makeup of an ecosystem. Different organisms prefer different salinities and substrates. Reconstruction of past environmental conditions is therefore possible by analyzing the abundance and distribution of remains of organisms preserved in shallow sediment cores collected throughout the region. This report will focus on the distribution of molluscan remains within a core collected from Whipray Basin in central Florida Bay.

\section{Setting}

Whipray Basin, located in north central Florida Bay (Figure 1), is approximately $11 \mathrm{mi}^{2}\left(17.6 \mathrm{~km}^{2}\right)$, and is surrounded by shallow mud banks and mangrove islands. The position and configuration of the basin restricts mixing and reduces the influence of tides and currents (Boyer et al., 1999), and can lead to hypersaline conditions and increased concentrations of organic material (McIvor, et al., 1994; Fourqurean, et al., 1992). From March 1991 through December 1999, monthly salinity ranged from 19.9 ppt to $52 \mathrm{ppt}$, with an average salinity of $34.1 \mathrm{ppt}^{1}$. Monthly temperature readings, taken from January 1997 through December 1998 , averaged $26.8^{\circ} \mathrm{C}$ and ranged from $10.1^{\circ} \mathrm{C}$ to $34.1^{\circ} \mathrm{C}$. Rainfall was monitored daily from August 1993 to December 1998 and ranged from no rainfall to an extreme of $7.19 \mathrm{~cm}$, with the average being $0.13 \mathrm{~cm}$ per day.

Whipray Basin is located just to the south of Rankin Lake, an area where very high salinities (> 50 ppt) were recorded between 1989-90 during a drought (Fourqurean, et al., 1992). The high salinity "bull's eye" documented for north-central Florida Bay in the late 80 's may be a typical annual pattern, although the actual salinity values will vary (McIvor, et al., 1994). Rankin Lake and other areas in west central and western Florida Bay were the sites of a massive seagrass mortality that began in 1987 and eventually led to the loss of more than 40,000 ha of seagrass (McIvor, et al., 1994; Robblee, et al., 1991). The proximity of the Whipray Basin to the hypersalinity bull's eye and the areas of extensive seagrass die-off, and the slow sedimentation rates within the basin, make it

\footnotetext{
${ }^{1}$ Everglades National Park, unpublished data, from water monitoring station located west of largest Whipray Key, close to core site.
} 


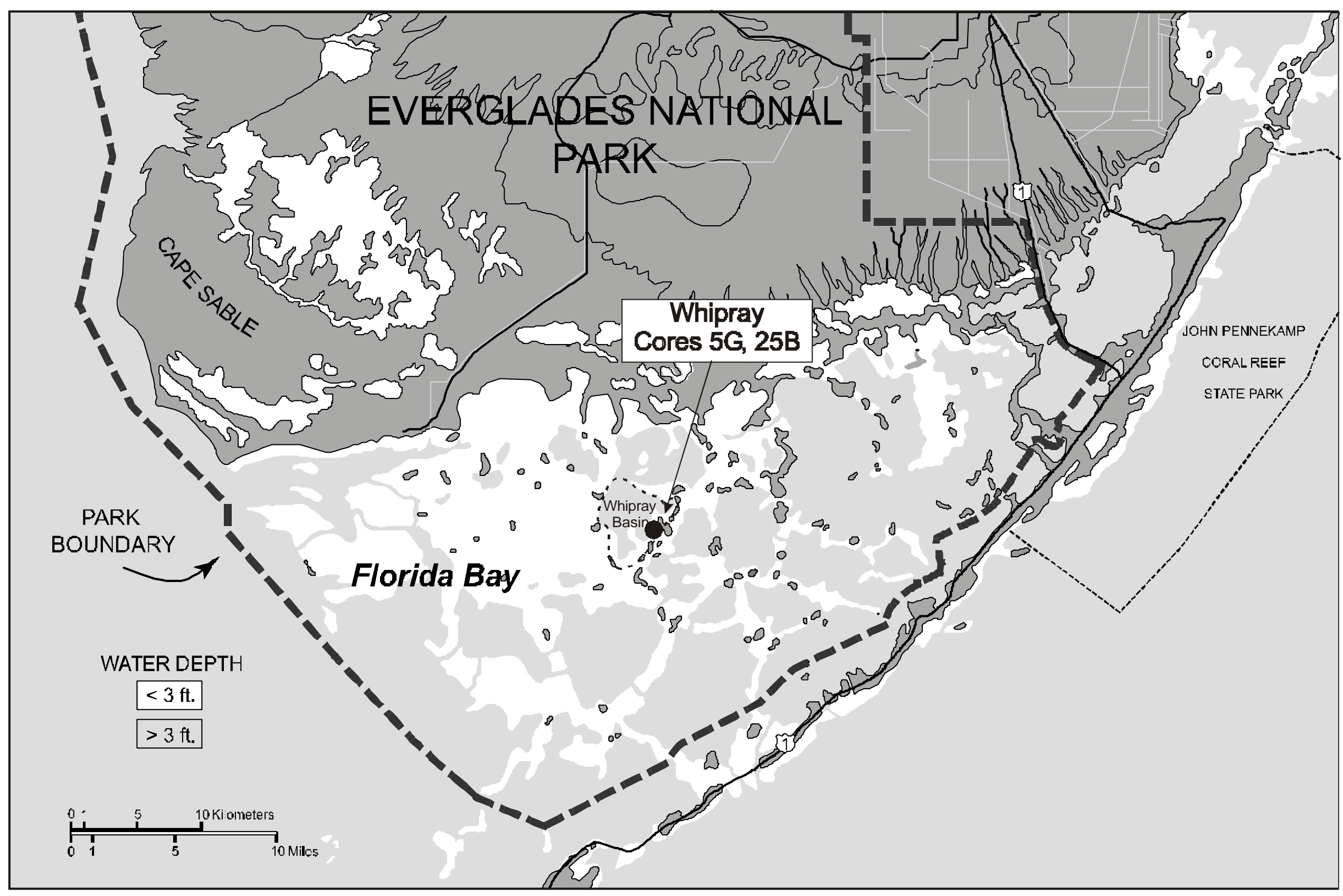

Figure 1: Location of Whipray Basin (dashed line) and cores 5G and 25B (solid circle) within Florida Bay. 
an ideal area for investigating ecosystem history. The results from this core will be contrasted to previous USGS Ecosystem History project studies from northern, eastern and east-central Florida Bay (Brewster-Wingard, et al., 1997, 1998a; 1998b, in press; Brewster-Wingard and Ishman, 1999; Ishman, et al., 1996; Wingard, et al., 1995) in future publications.

\section{ACKNOWLEDGEMENTS}

We would like to thank our colleagues at a number of other agencies, including South Florida Water Management District, National Oceanic and Atmospheric Administration, and Everglades National Park who have collaborated and cooperated on this research. We would especially like to thank the following: 1) the staff of Keys Marine Laboratory; all modern field work was done with their assistance; 2) Charles W. Holmes, U. S. Geological Survey, St. Petersburg, FL; he collected the core, and provide the age model; and 3) Angela Chong, South Florida Water Management District; she provided the modern water quality database, and assisted us in extracting the necessary data.

We would like to thank our reviewers, Tom Cronin and Bill Orem. Their thoughtful reviews have contributed to this paper. The following U. S. Geological Survey personnel have assisted in laboratory work and sample preparation: Marci Marot, Jill D'Ambrosio, and Jeffery Stone. The "Ecosystem History of Florida Bay and the Southwest Coast" project is a component of the U.S. Geological Survey's PlaceBased Studies Program. 


\section{METHODS}

\section{Core Collection}

Whipray core $25 \mathrm{~B}$, located at $\mathrm{N} 25.071258^{\circ}$, W $80.7385^{\circ}$ (Figure 1), was collected June 13, 1997 by Charles W. Holmes, U.S. Geological Survey, St. Petersburg, FL and his assistants. The four-inch diameter piston core penetrated $80 \mathrm{~cm}$ of shelly calcareous mud, and reached an underlying peat. The core was collected from a barren, non-grassy area. Two additional cores were collected in the same area for geochemical analyses.

\section{Processing and Analysis}

The core was sampled every two centimeters from the top down. Each sample was washed through a stack of $63 \mu \mathrm{m}$ and $850 \mu \mathrm{m}$ sieves. The sediment fraction less than $63 \mu \mathrm{m}$ was dried at $50{ }^{\circ} \mathrm{C}$ and weighed for ${ }^{210} \mathrm{~Pb}$ analysis. Any components greater than $63 \mu \mathrm{m}$ were dried at $50^{\circ} \mathrm{C}$ and separated for faunal analyses.

Every other sample (depths of 0-2 cm, 4-6 cm, etc.) was examined in detail. All identifiable molluscs were picked from each sample, identified, and counted (Table 1). These results are reported herein. Ostracodes and benthic foraminifera also were picked; these data will be reported separately. Mollusc identification was primarily determined using the taxonomy and descriptions of Abbott (1974), Warmke and Abbott (1961), and Perry and Schwengel (1955). All updated taxonomic nomenclature was based on Turgeon et al. (1998). In order to standardize the counts, data for each sample were converted to percent abundance data.

A cluster analysis (Figure 2) of the percent abundance data was performed using MVSP (Kovach Computing Services, MVSP Plus, version 3.1) following the methods described in Kovach $(1989,1995)$. The analysis was conducted with log-ratio transformed and centered data, then clustered using unweighted paired-group method, average-linkage, with cosine theta distance measurement, dual clustering procedure, and random input order. The cluster analysis was repeated several times in order to determine the accuracy of the clusters. A second cluster analysis excluding the rare species was conducted for comparison to the total data set; the results were the same.

\section{Age Model}

The age model for Whipray Basin is based on ${ }^{210} \mathrm{~Pb}$ and ${ }^{226} \mathrm{Ra}$ analyses from the less than $63 \mu \mathrm{m}$ fraction of the core samples. The results yielded a sedimentation rate of $0.43 \pm 0.07 \mathrm{~cm} / \mathrm{yr}$. (For a complete discussion of dating methods, see Holmes, et al., in press.) Table 2 shows the age/depth relations hip for core 25B. In addition, Whipray Core 5G, collected February 26, 1994 (N 25.0712, W 80.7385; Figure 1), has a sedimentation rate of $0.42 \pm 0.04 \mathrm{~cm} / \mathrm{yr}$. The similarity in sedimentation rates between the two cores indicates the stratigraphy is consistent at the coring sites within Whipray Basin. 
Table 1: Percent abundance of molluscan species, total abundance, and faunal richness in samples from Whipray core 25B.

\begin{tabular}{|c|c|c|c|c|c|c|c|c|c|c|c|c|c|c|c|c|c|c|c|c|c|}
\hline Species & $0-2$ & $4-6$ & $8-10$ & $2-14$ & $16-18$ & $20-22$ & $24-26$ & $28-30$ & $32-34$ & 36-38 & $40-42$ & 44-46 & $48-50$ & $52-54$ & $56-58$ & $60-62$ & 64-66 & $8-70$ & $72-74$ & 76-78 & 78-80 \\
\hline Acteocina canaliculata & 22.22 & 12.50 & 0.00 & 0.00 & 0.00 & 0.60 & 0.14 & 0.45 & 0.15 & 0.92 & 0.32 & 0.00 & 0.00 & 0.00 & 0.72 & 0.39 & 0.61 & 0.36 & 0.00 & 0.22 & 0.00 \\
\hline nomalocardia auberiana & 0.00 & 0.00 & 7.14 & 39.06 & 1.68 & 2.40 & 1.57 & 0.00 & & & & 0.00 & & & & & 0.49 & & & 1.52 & 0.00 \\
\hline Arcopsis adamsi & 0.00 & 0.00 & 0.00 & 0.00 & 0.00 & & & 0.00 & 0.00 & & 0.00 & 0.00 & 0.00 & & 0.00 & & 0.49 & & & 0.65 & 0.00 \\
\hline Argopecten irradians & 0.00 & 0.00 & 0.00 & 0.00 & 0.00 & 0.00 & 0.14 & 0.00 & 0.00 & 0.00 & 0.00 & 0.00 & 0.00 & 0.57 & 0.00 & 0.26 & 0.24 & 0.72 & 0.41 & 0.22 & 0.00 \\
\hline Batillaria minima & 0.00 & 0.00 & 0.00 & 0.00 & 0.00 & 0.00 & 0.00 & 0.00 & 0.00 & 0.00 & 0.00 & 0.00 & 0.00 & 0.00 & 0.00 & 0.00 & 0.12 & 0.00 & 0.62 & 0.00 & 0.00 \\
\hline Bittiolum varium & 11.11 & 12.50 & 0.00 & 0.00 & 1.68 & 3.40 & 3.43 & 6.00 & 4.46 & 3.92 & 1.62 & 1.38 & 2.38 & 6.52 & 10.87 & 22.63 & 24.02 & 13.54 & 15.20 & 14.57 & 0.00 \\
\hline Boonea impressa & 0.00 & 0.00 & 0.00 & 0.00 & 0.84 & 0.00 & 0.00 & 0.00 & 0.00 & 0.00 & 0.00 & 0.00 & & 0.00 & 0.00 & 0.00 & 0.00 & 0.00 & 0.00 & 0.00 & 0.00 \\
\hline Brachidontes & 33.33 & 25.00 & 78.57 & 26.56 & 43.70 & 34.40 & 29.43 & 23.69 & 25.26 & 75 & 28.53 & 41.01 & & 35.69 & 35.87 & 19.47 & 18.90 & 20.76 & & 21.09 & 34.78 \\
\hline Bulla striata & 0.00 & 0.00 & 0.00 & 2.34 & 3.36 & 2.20 & 3.14 & 2.85 & 2.23 & & .24 & 1.38 & & 0.28 & 0.36 & & 1.59 & 0.18 & & 0.22 & 0.00 \\
\hline cornucopiae & .00 & 0.00 & 0.00 & 0.00 & 0.00 & & 0.00 & 0.00 & .15 & & .00 & 0.00 & & 0.00 & 0.00 & 0.00 & 0.37 & .36 & 0.00 & 0.00 & 0.00 \\
\hline ulchellum & 0.00 & 0.00 & 0.00 & 0.00 & 0.00 & & 0.29 & & & & 1.46 & & & 0. & 0.36 & 0.39 & & .36 & 0.21 & 1.30 & 0.00 \\
\hline floridana & .00 & 0.00 & .00 & 0.00 & .00 & & 0.00 & .00 & & & .00 & & & & & & & & & 0.22 & 0.00 \\
\hline stata & 00 & 0.00 & 0.00 & 0.00 & 0.00 & & 0.00 & & 0.00 & & .00 & & & & 0.00 & & 0.00 & & & 0.22 & 0.00 \\
\hline Cerithiop & 0.00 & 0.00 & & 0.00 & & & & & & & & & & & & & & & 0 & 0.00 & 0.00 \\
\hline neum? & .00 & 0.00 & 0.00 & 0.00 & 0.00 & & 0.00 & & & & 0.00 & & & & 0.00 & & & & & 65 & 0.00 \\
\hline & 0.00 & 12.50 & 7.14 & 3.91 & 5.04 & & 5.71 & & 6.84 & & 6.81 & 7.83 & & & 7.97 & & 12.32 & & 16.22 & 23.91 & 17.39 \\
\hline & 0.00 & & & & & & & & & & & & & & & & & & & & 0.00 \\
\hline & 00 & 0.00 & .00 & 0.00 & 0.00 & & 0.29 & & & & & & & & & & & & & 0.00 & 0.00 \\
\hline Chic & 00 & 0.00 & 0.00 & 0.00 & & & & & & & 1.46 & & & & 0.72 & 2.3 & 4.02 & & & 2.83 & 4.35 \\
\hline & & & & & & & & & & & & & & & & & & & & & 0.00 \\
\hline & 00 & 0.00 & 0.00 & 0.00 & 0.0 & & 0.0 & & $0 .($ & & & & & & & & & & & 00 & 0.00 \\
\hline , & 0 & 0.00 & & 0.00 & & & & & & & & & & & & & & & & & 0.00 \\
\hline & 5.56 & 0.00 & & & 11.7 & & 17.57 & 17. & & & & & & & & & & & & & 13.04 \\
\hline inoides & 0.00 & 0.00 & & & & & & & & & & & & & & & & & & 0.22 & 0.00 \\
\hline & 0.00 & & & & & & & & & & & & & & & & & & & & 0.00 \\
\hline Der & 0.00 & 0.00 & & & & & & & & & & & & & & & & & & & 0.00 \\
\hline Der & 0.00 & & & & & & & & & & & & & & & & & & & & 0.00 \\
\hline & 0.00 & 0.00 & & & & & & & & & & & & & & & 0.0 & & $0 .($ & 0. & 0.00 \\
\hline Dioa & 0.00 & 0.00 & 0.00 & 0.00 & 0.00 & 0.00 & 0.00 & 0.00 & 0.00 & 0.0 & 0.00 & 0.00 & 0.0 & 0.0 & 0.36 & 0.0 & 0.00 & 18 & $0 .($ & 0.00 & 0.00 \\
\hline & & & & & & & & & & & & & & & & & & & & & 0.00 \\
\hline Euli & 0.00 & 0.00 & & & & & & & & & & & & & & & & & $0 .($ & 0.00 & 0.00 \\
\hline hunteri & 0.00 & 0.00 & 7.14 & 0.00 & 0.00 & 0.00 & 0.00 & 0.00 & 0.00 & & 0.00 & 0.00 & & 0.0 & 0.00 & 0.0 & 0.00 & & 0 & 0.00 & 0.00 \\
\hline Finella & & & & & & & & & & & & & & & & & & & & & 0.00 \\
\hline & & & & & & & & & & & & & & & & & & & & & 0.00 \\
\hline & & 0.00 & & & 0.00 & & & & & & & & & & & & & & & & 0.00 \\
\hline & & 0.00 & & & & & & & & & & & & & 0.72 & & 1. & & & & 0.00 \\
\hline & & & & & & & & & & & & & & & & & & & & & 0.00 \\
\hline Latirus sp.? & 0.00 & 0.00 & & & 0.00 & & 0.00 & & & & & 0.00 & & & 0.00 & & & .00 & & 0.00 & 0.00 \\
\hline & 0.00 & 0.00 & & & & & 0.00 & & & & & & & 0.00 & 0.00 & 0.00 & 0.00 & 0.00 & 0.00 & 0.00 & 0.00 \\
\hline Limaria sp. cf. L. pellucida & 0.00 & & & & & & & & & & & & & & & & & & & & 0.00 \\
\hline Longchaeus crenulatus? & 0.00 & 0.00 & 0.00 & 0.00 & 0.00 & & 0.00 & 0.15 & 0.00 & 0.00 & 0.00 & 0.00 & 0.00 & 0.00 & 0.00 & 0.00 & 0.00 & 0.00 & 0.00 & 0.00 & 0.00 \\
\hline Lucinisca cf. nassula & 0.00 & 0.00 & & 0.00 & & & & & & & & & & & 0.00 & & 0.12 & 0.00 & 0.00 & 0.00 & 0.00 \\
\hline Lucinisca nassula & 0.00 & 0.00 & 0.00 & 0.00 & 0.00 & 0.00 & 0.00 & 0.00 & 0.00 & 0.00 & 0.16 & 0.00 & 0.00 & 0.28 & 0.72 & 0.79 & 0.24 & 0.00 & 0.62 & 0.65 & 0.00 \\
\hline
\end{tabular}


Table 1: Percent abundance of molluscan species, total abundance, and faunal richness in samples from Whipray core 25B.

\begin{tabular}{|c|c|c|c|c|c|c|c|c|c|c|c|c|c|c|c|c|c|c|c|c|c|}
\hline $\begin{array}{lll}\text { Species } & \text { Sample Depth } \\
\end{array}$ & $0-2$ & 4-6 & $8-10$ & $12-14$ & $16-18$ & $20-22$ & $24-26$ & 28-30 & $32-34$ & $36-38$ & $40-42$ & $44-46$ & $48-50$ & $52-54$ & $56-58$ & $60-62$ & 64-66 & $68-70$ & $72-74$ & $76-78$ & $78-80$ \\
\hline Marginellidae & 0.00 & 0.00 & 0.00 & 0.00 & 0.00 & 0.00 & 0.14 & 0.00 & 0.00 & 0.00 & 0.00 & 0.00 & 0.00 & 0.00 & 0.00 & 0.00 & 0.00 & 0.00 & 0.00 & 0.00 & 0.00 \\
\hline Marshallora nigrocincta & 0.00 & 0.00 & 0.00 & 0.00 & 0.00 & 0.40 & 0.14 & 0.00 & 0.30 & 0.00 & 0.00 & 0.00 & 0.00 & 0.00 & 0.00 & 0.00 & 0.00 & 0.00 & 0.00 & 0.00 & 0.00 \\
\hline Modulus modulus & 0.00 & 0.00 & 0.00 & 1.56 & 0.84 & 0.60 & 1.43 & 1.95 & 1.34 & 2.00 & 1.30 & 1.38 & 1.19 & 0.85 & 0.72 & 3.55 & 1.95 & 1.44 & 5.13 & 3.26 & 4.35 \\
\hline Mysella sp.? & 0.00 & 0.00 & 0.00 & 0.00 & 0.00 & 0.00 & 0.14 & 0.00 & 0.30 & 0.00 & 0.00 & 0.00 & 0.00 & 0.00 & 0.00 & 0.00 & 0.12 & 0.00 & 0.00 & 0.00 & 0.00 \\
\hline Nassarius sp. & 0.00 & 0.00 & 0.00 & 0.00 & 0.00 & 0.00 & 0.14 & 0.15 & 0.00 & 0.08 & 0.00 & 0.00 & 0.00 & 0.00 & 0.00 & 0.00 & 0.00 & 0.00 & 0.00 & 0.00 & 0.00 \\
\hline Nigrocincta sp. & 0.00 & 0.00 & 0.00 & 0.00 & 0.00 & 0.00 & 0.00 & 0.00 & 0.00 & 0.00 & 0.00 & 0.00 & 0.00 & 0.00 & 0.00 & 0.13 & 0.00 & 0.00 & 0.00 & 0.00 & 0.00 \\
\hline Nucula proxima & 0.00 & 0.00 & 0.00 & 0.00 & 0.00 & 0.40 & 0.00 & 0.00 & 0.00 & 0.00 & 0.32 & 0.00 & 0.00 & 0.00 & 0.00 & 0.26 & 0.73 & 0.90 & 1.64 & 1.96 & 0.00 \\
\hline Odostomia canaliculata? & 0.00 & 0.00 & 0.00 & 0.00 & 0.00 & 0.00 & 0.00 & 0.00 & 0.00 & 0.00 & 0.00 & 0.00 & 0.00 & 0.00 & 0.00 & 0.00 & 0.00 & 0.00 & 0.21 & 0.00 & 0.00 \\
\hline Odostomia laevigata & 0.00 & 0.00 & 0.00 & 0.00 & 0.00 & 0.20 & 0.57 & 0.15 & 0.15 & 0.92 & 0.00 & 0.00 & 0.00 & 0.00 & 0.00 & 1.18 & 1.22 & 0.18 & 0.00 & 0.22 & 0.00 \\
\hline Odostomia sp.? & 0.00 & 0.00 & 0.00 & 0.00 & 0.00 & 0.00 & 0.00 & 0.30 & 0.00 & 0.00 & 0.00 & 0.00 & 0.00 & 0.00 & 0.00 & 0.00 & 0.00 & 0.00 & 0.00 & 0.00 & 0.00 \\
\hline Olivella pusilla & 5.56 & 0.00 & 0.00 & 0.78 & 0.84 & 0.60 & 1.14 & 1.05 & 1.63 & 1.42 & 0.81 & 0.46 & 0.00 & 0.57 & 0.00 & 0.53 & 0.98 & 0.54 & 0.41 & 0.65 & 0.00 \\
\hline Opercula - chitonous & 0.00 & 0.00 & 0.00 & 0.78 & 0.00 & 0.00 & 0.00 & 0.00 & 0.00 & 0.00 & 0.00 & 0.00 & 0.00 & 0.00 & 0.00 & 0.00 & 0.00 & 0.00 & 0.00 & 0.00 & 0.00 \\
\hline Parastarte triquetra & 0.00 & 0.00 & 0.00 & 0.00 & 0.00 & 1.40 & 0.86 & 1.20 & 2.08 & 4.08 & 0.81 & 0.46 & 0.00 & 0.28 & 0.72 & 0.66 & 0.85 & 1.81 & 0.21 & 1.09 & 0.00 \\
\hline Parvilucina multilineata & 0.00 & 0.00 & 0.00 & 0.00 & 0.00 & 0.00 & 0.00 & 0.00 & 0.00 & 0.00 & 0.00 & 0.00 & 0.00 & 0.00 & 0.00 & 0.00 & 0.12 & 0.00 & 0.00 & 0.00 & 0.00 \\
\hline Pilsbryspira leucocyma & 0.00 & 0.00 & 0.00 & 0.00 & 0.00 & 0.00 & 0.14 & 0.00 & 0.15 & 0.17 & 0.32 & 0.00 & 0.00 & 0.28 & 0.00 & 0.00 & 0.00 & 0.18 & 0.21 & 0.00 & 0.00 \\
\hline Pitar simpsoni & 0.00 & 0.00 & 0.00 & 0.00 & 0.00 & 0.00 & 0.00 & 0.00 & 0.00 & 0.33 & 0.00 & 0.00 & 0.00 & 0.00 & 0.00 & 0.00 & 0.24 & 0.00 & 0.00 & 0.00 & 0.00 \\
\hline Prunum apicinum & 5.56 & 0.00 & 0.00 & 7.03 & 2.52 & 1.20 & 1.00 & 1.50 & 1.19 & 1.00 & 1.62 & 1.38 & 1.19 & 0.85 & 0.00 & 0.66 & 1.34 & 0.00 & 0.41 & 0.22 & 0.00 \\
\hline Pteria longisquamosa & 0.00 & 0.00 & 0.00 & 0.00 & 3.36 & 0.40 & 0.43 & 0.75 & 2.08 & 1.00 & 0.97 & 0.46 & 0.00 & 0.57 & 2.54 & 1.71 & 1.34 & 1.08 & 1.64 & 0.43 & 0.00 \\
\hline Rictaxis punctostriatus & 0.00 & 0.00 & 0.00 & 0.00 & 0.00 & 0.20 & 0.00 & 0.15 & 0.00 & 0.00 & 0.00 & 0.00 & 0.00 & 0.00 & 0.00 & 0.00 & 0.00 & 0.00 & 0.00 & 0.00 & 0.00 \\
\hline Rissoina cancellata & 0.00 & 0.00 & 0.00 & 0.00 & 0.00 & 0.20 & 0.29 & 0.15 & 0.15 & 0.25 & 0.16 & 1.84 & 0.00 & 1.42 & 0.36 & 1.18 & 0.24 & 0.90 & 1.03 & 1.30 & 0.00 \\
\hline Schwartziella catesbyana & 0.00 & 12.50 & 0.00 & 0.78 & 1.68 & 1.20 & 2.00 & 1.80 & 1.63 & 2.75 & 2.76 & 3.69 & 1.19 & 2.83 & 3.62 & 2.50 & 4.51 & 5.23 & 5.34 & 5.22 & 8.70 \\
\hline Stellatoma stellata & 0.00 & 0.00 & 0.00 & 0.00 & 0.00 & 0.40 & 0.00 & 0.00 & 0.00 & 0.00 & 0.00 & 0.00 & 0.00 & 0.00 & 0.00 & 0.79 & 0.00 & 0.36 & 1.03 & 0.43 & 0.00 \\
\hline Teinostoma sp. & 0.00 & 0.00 & 0.00 & 0.00 & 0.00 & 0.00 & 0.00 & 0.00 & 0.00 & 0.00 & 0.00 & 0.00 & 0.00 & 0.00 & 0.00 & 0.00 & 0.00 & 0.18 & 0.00 & 0.00 & 0.00 \\
\hline Tellina spp. & 0.00 & 0.00 & 0.00 & 1.56 & 0.00 & 0.20 & 0.43 & 0.30 & 0.45 & 0.33 & 0.16 & 0.46 & 0.00 & 0.57 & 0.00 & 0.79 & 0.12 & 0.90 & 0.41 & 0.43 & 0.00 \\
\hline Transennella sp. & 5.56 & 12.50 & 0.00 & 7.81 & 14.29 & 28.20 & 25.86 & 30.88 & 26.00 & 32.17 & 30.63 & 14.29 & 52.38 & 27.76 & 19.57 & 9.47 & 7.20 & 13.54 & 10.68 & 6.52 & 4.35 \\
\hline Truncatella sp. & 0.00 & 0.00 & 0.00 & 0.00 & 0.00 & 0.00 & 0.00 & 0.00 & 0.00 & 0.00 & 0.00 & 0.00 & 0.00 & 0.00 & 0.00 & 0.00 & 0.00 & 0.00 & 0.00 & 0.22 & 0.00 \\
\hline Turbonilla sp. & 0.00 & 0.00 & 0.00 & 0.00 & 0.00 & 0.00 & 0.00 & 0.00 & 0.00 & 0.00 & 0.00 & 0.00 & 0.00 & 0.00 & 0.00 & 0.13 & 0.37 & 0.18 & 0.21 & 0.00 & 0.00 \\
\hline Turridae & 0.00 & 0.00 & 0.00 & 0.00 & 0.00 & 0.00 & 0.00 & 0.00 & 0.30 & 0.00 & 0.16 & 0.00 & 0.00 & 0.00 & 0.00 & 0.13 & 0.00 & 0.00 & 0.21 & 0.00 & 0.00 \\
\hline Unidentified Gastropods & 5.56 & 0.00 & 0.00 & 0.00 & 1.68 & 0.40 & 0.43 & 0.30 & 0.00 & 0.25 & 0.00 & 0.00 & 0.00 & 0.28 & 1.45 & 0.13 & 1.59 & 0.36 & 0.21 & 0.65 & 0.00 \\
\hline Unidentified Pelecypods & 0.00 & 12.50 & 0.00 & 0.00 & 3.36 & 0.00 & 0.00 & 0.00 & 0.00 & 0.00 & 0.00 & 0.46 & 0.00 & 0.28 & 0.00 & 0.00 & 0.12 & 1.26 & 0.21 & 0.22 & 8.70 \\
\hline Vermicularia sp. & 0.00 & 0.00 & 0.00 & 0.00 & 0.00 & 0.00 & 0.00 & 0.00 & 0.00 & 0.00 & 0.00 & 0.00 & 0.00 & 0.00 & 0.00 & 0.00 & 0.00 & 0.00 & 0.21 & 0.87 & 0.00 \\
\hline Vitrinellidae & 0.00 & 0.00 & 0.00 & 0.00 & 0.00 & 0.00 & 0.00 & 0.00 & 0.00 & 0.00 & 0.00 & 0.00 & 0.00 & 0.00 & 0.00 & 0.00 & 0.00 & 0.00 & 0.21 & 0.00 & 4.35 \\
\hline Vitrinella floridana & 0.00 & 0.00 & 0.00 & 0.00 & 0.00 & 0.00 & 0.00 & 0.15 & 0.00 & 0.08 & 0.00 & 0.00 & 0.00 & 0.00 & 0.00 & 0.00 & 0.00 & 0.00 & 0.00 & 0.00 & 0.00 \\
\hline $\begin{array}{l}\text { Total no. of Faunal } \\
\text { Groups/sample } \\
\text { (Faunal Richness) }\end{array}$ & 9 & 7 & 4 & 13 & 17 & 28 & 33 & 30 & 31 & 33 & 29 & 18 & 9 & 27 & 22 & 41 & 39 & 36 & 36 & 36 & 9 \\
\hline
\end{tabular}


Figure 2: Q-mode cluster diagram using MVSP program. Numbers on right-hand axis are sample depths in $\mathrm{cm}$. See text for details.

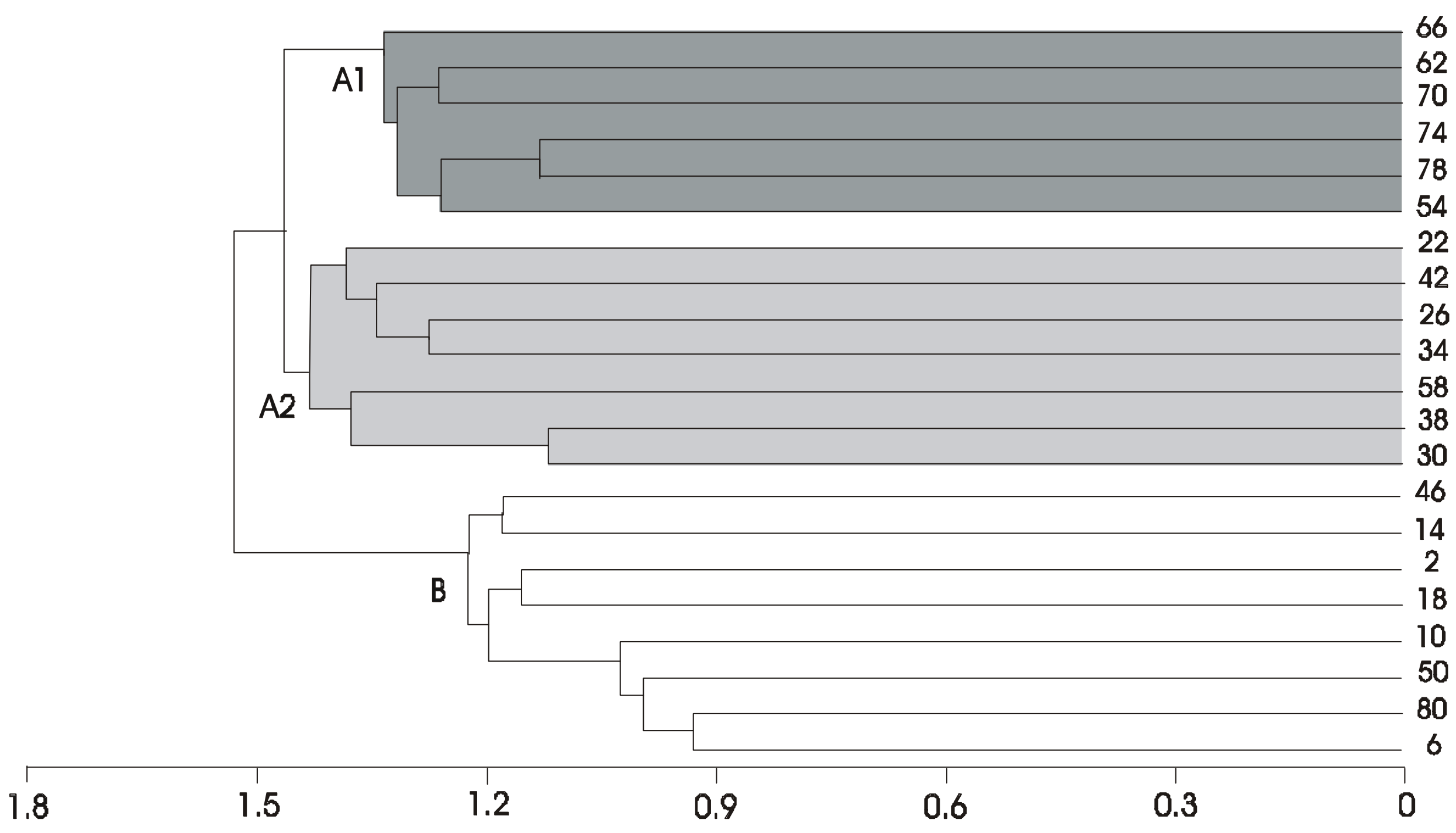

Cosine Theta - Data Centered to log ratio transformed 
Table 2: Age-Depth relationship for samples from Whipray core 25B. Sedimentation rate, based on ${ }^{210} \mathrm{~Pb}$ analysis is $0.43 \mathrm{~cm} / \mathrm{yr}+/-0.07$. Core was collected in 1997 .

\begin{tabular}{|c|c|c|c|c|c|c|c|}
\hline Depth $(\mathrm{cm})$ & $\begin{array}{l}\text { Years before } \\
\text { present (ybp) }\end{array}$ & $\begin{array}{c}\text { Average } \\
\text { Year }\end{array}$ & $\begin{array}{c}\text { Positive error } \\
(\mathrm{ybp})\end{array}$ & $\begin{array}{c}\text { Maximum } \\
\text { year }\end{array}$ & $\begin{array}{c}\text { Negative } \\
\text { error (ybp) }\end{array}$ & $\begin{array}{c}\text { Minimum } \\
\text { Year }\end{array}$ & Range of Years \\
\hline 2 & 4.66 & 1992 & 4.00 & 1993 & 5.72 & 1991 & 1991-1993 \\
\hline 4 & 9.32 & 1988 & 8.00 & 1989 & 11.44 & 1986 & 1985-1989 \\
\hline 6 & 13.98 & 1983 & 12.00 & 1985 & 17.16 & 1980 & 1979-1985 \\
\hline 8 & 18.64 & 1978 & 16.00 & 1981 & 22.88 & 1974 & 1974-1981 \\
\hline 10 & 23.30 & 1974 & 20.00 & 1977 & 28.60 & 1968 & 1968-1977 \\
\hline 12 & 27.96 & 1969 & 24.00 & 1973 & 34.32 & 1963 & $1962-1973$ \\
\hline 14 & 32.62 & 1964 & 28.00 & 1969 & 40.04 & 1957 & 1956-1969 \\
\hline 16 & 37.28 & 1960 & 32.00 & 1965 & 45.76 & 1951 & $1951-1965$ \\
\hline 18 & 41.94 & 1955 & 36.00 & 1961 & 51.48 & 1946 & $1945-1961$ \\
\hline 20 & 46.60 & 1950 & 40.00 & 1957 & 57.20 & 1940 & 1939-1957 \\
\hline 22 & 51.26 & 1946 & 44.00 & 1953 & 62.92 & 1934 & 1934-1953 \\
\hline 24 & 55.92 & 1941 & 48.00 & 1949 & 68.64 & 1928 & 1928-1949 \\
\hline 26 & 60.58 & 1936 & 52.00 & 1945 & 74.36 & 1923 & $1922-1945$ \\
\hline 28 & 65.24 & 1932 & 56.00 & 1941 & 80.08 & 1917 & 1916-1941 \\
\hline 30 & 69.90 & 1927 & 60.00 & 1937 & 85.80 & 1911 & 1911-1937 \\
\hline 32 & 74.56 & 1922 & 64.00 & 1933 & 91.52 & 1905 & $1905-1933$ \\
\hline 34 & 79.22 & 1918 & 68.00 & 1929 & 97.24 & 1900 & 1899-1929 \\
\hline 36 & 83.88 & 1913 & 72.00 & 1925 & 102.96 & 1894 & 1894-1925 \\
\hline 38 & 88.54 & 1908 & 76.00 & 1921 & 108.68 & 1888 & 1888-1921 \\
\hline 40 & 93.20 & 1904 & 80.00 & 1917 & 114.40 & 1883 & $1882-1917$ \\
\hline 42 & 97.86 & 1899 & 84.00 & 1913 & 120.12 & 1877 & 1876-1913 \\
\hline 44 & 102.52 & 1894 & 88.00 & 1909 & 125.84 & 1871 & 1871-1909 \\
\hline 46 & 107.18 & 1890 & 92.00 & 1905 & 131.56 & 1865 & $1865-1905$ \\
\hline 48 & 111.84 & 1885 & 96.00 & 1901 & 137.28 & 1860 & 1859-1901 \\
\hline 50 & 116.50 & 1881 & 100.00 & 1897 & 143.00 & 1854 & 1854-1897 \\
\hline 52 & 121.16 & 1876 & 104.00 & 1893 & 148.72 & 1848 & $1848-1893$ \\
\hline 54 & 125.82 & 1871 & 108.00 & 1889 & 154.44 & 1843 & $1842-1889$ \\
\hline 56 & 130.48 & 1867 & 112.00 & 1885 & 160.16 & 1837 & $1836-1885$ \\
\hline 58 & 135.14 & 1862 & 116.00 & 1881 & 165.88 & 1831 & $1831-1881$ \\
\hline 60 & 139.80 & 1857 & 120.00 & 1877 & 171.60 & 1825 & $1825-1877$ \\
\hline 62 & 144.46 & 1853 & 124.00 & 1873 & 177.32 & 1820 & $1819-1873$ \\
\hline 64 & 149.12 & 1848 & 128.00 & 1869 & 183.04 & 1814 & 1813-1869 \\
\hline 66 & 153.78 & 1843 & 132.00 & 1865 & 188.76 & 1808 & $1808-1865$ \\
\hline 68 & 158.44 & 1839 & 136.00 & 1861 & 194.48 & 1803 & $1802-1861$ \\
\hline 70 & 163.10 & 1834 & 140.00 & 1857 & 200.20 & 1797 & 1796-1857 \\
\hline 72 & 167.76 & 1829 & 144.00 & 1853 & 205.92 & 1791 & $1791-1853$ \\
\hline 74 & 172.42 & 1825 & 148.00 & 1849 & 211.64 & 1785 & $1785-1849$ \\
\hline 76 & 177.08 & 1820 & 152.00 & 1845 & 217.36 & 1780 & $1779-1845$ \\
\hline 78 & 181.74 & 1815 & 156.00 & 1841 & 223.08 & 1774 & $1773-1841$ \\
\hline 80 & 186.40 & 1811 & 160.00 & 1837 & 228.80 & 1768 & $1768-1837$ \\
\hline
\end{tabular}




\section{RESULTS}

The compiled data from the molluscan counts in each sample revealed the presence of 78 faunal groups $^{2}$ (Table 1). Of these groups, there are five that dominate the core, accounting for $76 \%$ of the individuals (Figure 3). Four of the dominant groups are epifaunal; these are Brachidontes exustus, Crepidula spp., Cerithium muscarum, and Bittiolum varium. Transennella sp. is the only dominant infaunal group. Throughout the core, faunal richness ${ }^{3}$ and abundance are highly variable. Faunal richness ranges from 9 to 41 , yielding an average of 24 groups per sample. The average number of specimens per sample is 413, with a range from 8 to 1200.

Cluster analysis revealed the presence of 3 clusters within the core (Figure 2). These clusters separate the core into zones 1,3, and 4, with transition zone 2 from 58 to $44 \mathrm{~cm}$. The samples from the transition zone occur in all 3 clusters in the analysis. Clusters A1 and A2 make up the majority of the core. They are characterized by high faunal richness and abundance and are separated by the presence or absence of a few rare species. The rare species were included in the analyses because we believe they contain valuable information about subtle changes in the environment. Cluster B represents samples with low faunal richness and abundance and includes the top of the core as well as samples at 46, 50, and $80 \mathrm{~cm}$. The sample at $80 \mathrm{~cm}$ is distinct from the overlying samples in zone 1 , and is most like the samples in zone 4 at the top of the core.

Zone 1, from 78-60 centimeters, constitutes a zone of high faunal richness and abundance, and is represented by Cluster A1 (Figure 2, Figure 3). Although the average faunal richness is 38 and the average abundance is 616 specimens, these values fluctuate somewhat throughout this lower zone. The most dominant faunal group is Brachidontes exustus, followed by Bittiolum varium, Cerithium muscarum, and Transennella sp. (Figure 3, Table 1). The lowest section of this zone $(78-68 \mathrm{~cm})$ contains the low-salinity species Truncatella sp., Polymesoda maritima, and Cerithidea costata as well as freshwater Hydrobiidae. Bittiolum varium abundance peaks at $66 \mathrm{~cm}$ and then decreases and remains low until the upper $10 \mathrm{~cm}$ where it increases slightly. While Cerithium muscarum is a dominant species throughout the core, its abundance is much higher in zone 1 , than in the other zones.

Zone 2, from 58-44 cm, constitutes a transition zone. Consequently, samples from this zone are found in all three clusters (Figure 2). The samples from 58 to $52 \mathrm{~cm}$ signify the beginning of a drop in faunal richness and abundance (Figure 3, Table 1). The average number of individuals decreases by half to 314.5 and faunal richness falls to 24.5. This drop is due to the absence of a number of rare species that had been present in zone 1. Brachidontes exustus and Transennella sp. remain dominant species. The samples from the upper part of the transition zone, 50 to $44 \mathrm{~cm}$, are included in Cluster B.

\footnotetext{
${ }^{2}$ Faunal groups are usually species, but may be genera, or occasionally broader categories (eg. Marginellidae).

${ }^{3}$ Faunal richness in this study is a measure of the number of faunal groups present in a given sample so this is not "species" richness in the usual sense. It combines species, generic, and occasionally higher taxonomic level data.
} 
Figure 3: Plots showing the percent abundance of select species down-core, the distribution of clusters (see Figure 2) within the core, and total abundance (total number of molluscan specimens) and faunal richness (total number of groups identified) for each sample.

Depth in $\mathrm{cm}$ is plotted on the left, year and zone on the right. Rainfall data is a compilation of NOAA divisions 5, 6, and 7 rainfall data.

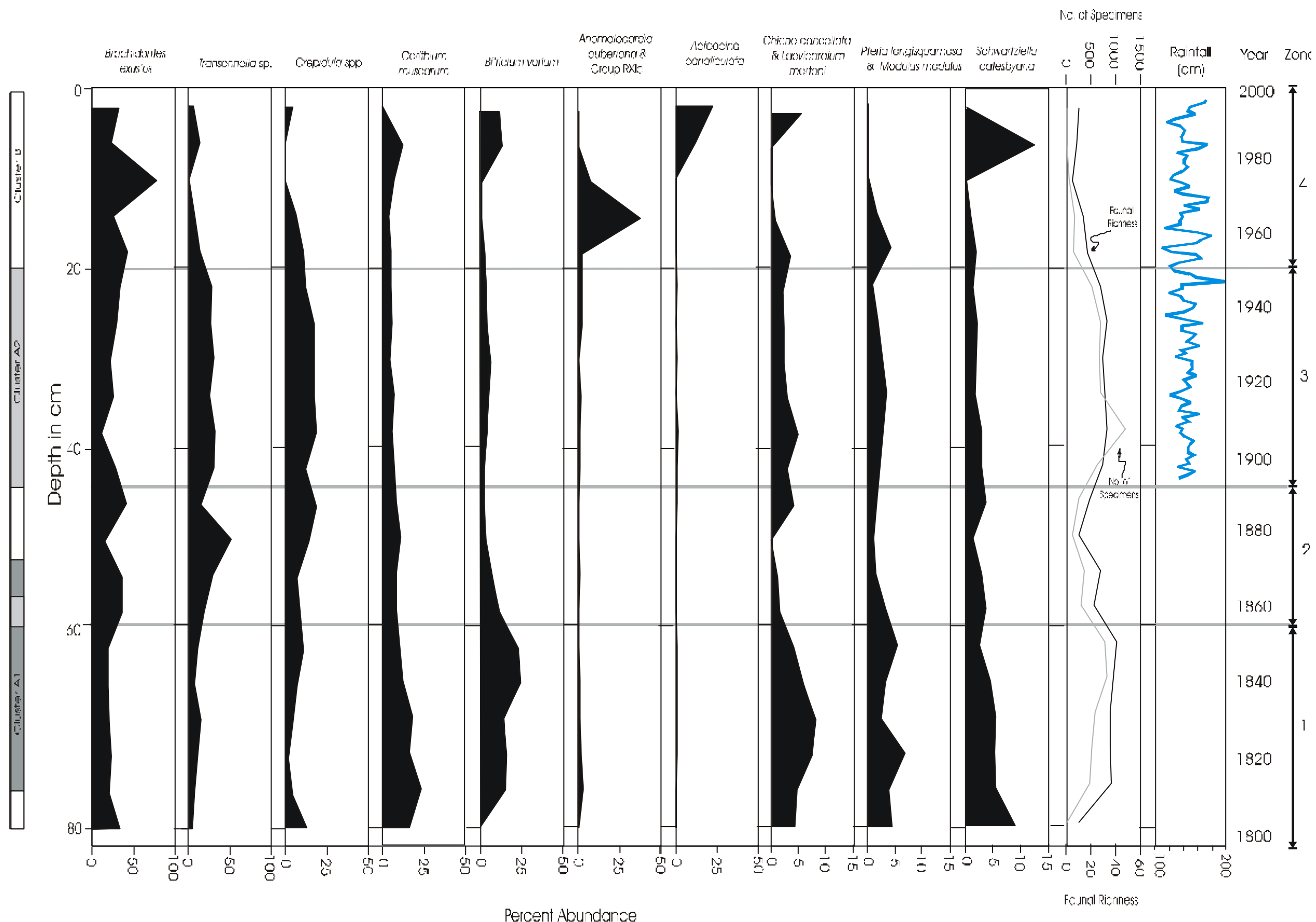


Both the measures of diversity and the patterns of dominance undergo notable changes. Faunal richness and abundance experience a significant drop followed by an increase. Moving up the core from $50 \mathrm{~cm}$ to $44 \mathrm{~cm}$, faunal richness (9 to 18) and abundance (84 to 217) double compared to the underlying samples. Transennella sp. dominates the $50 \mathrm{~cm}$ sample, while Brachidontes exustus dominates the $46 \mathrm{~cm}$ sample. Crepidula spp. and Cerithium muscarum also make up a significant part of this section, but their abundance is fairly constant.

Zone $3(42-20 \mathrm{~cm})$ is dominated by Transennella sp., followed closely by Brachidontes exustus (Figure 3). Other species with a significant presence are Crepidula spp. and Cerithium muscarum. The samples from zone 3 fall in Cluster A2 (Figure 2). Measures of diversity are high and stable throughout this section of the core, with all samples having an abundance value near 726 and an average faunal richness of 31 . The one exception is a peak in abundance (1200 specimens) for the $38 \mathrm{~cm}$ sample.

Zone 4, the top of the core from 18 to $0 \mathrm{~cm}$, is overwhelmingly dominated by Brachidontes exustus (Figure 3, Table 1). However, Anomalocardia auberiana, Transennella sp., Acteocina canaliculata, and Cerithium muscarum also are present in significant numbers in this zone. The presence of Anomalocardia auberiana and Acteocina canaliculata is significant because they do not appear in great abundance anywhere else in the core. Measures of diversity are the lowest in this section of the core, with an average abundance of 57 species per sample and an average faunal richness of 10. Samples from zone 4 occur in Cluster B (Figure 2).

\section{DISCUSSION}

Analysis of molluscan assemblages provides a model for Whipray Basin's environment throughout the past 200 years. The sedimentation rate in Whipray Basin is low $(0.43 \mathrm{~cm} / \mathrm{yr})$ compared to other cores examined from Russell Bank $(1.27 \mathrm{~cm} / \mathrm{yr})$, Bob Allen mudbank $(0.75 \mathrm{~cm} / \mathrm{yr})$, and Pass Key $(3.5 \mathrm{~cm} / \mathrm{yr})$ (Holmes et al., in press). Each 2$\mathrm{cm}$ sampling interval in Whipray core 25B represents 4.7 years of deposition so shortterm seasonal trends cannot be detected. The changes that are discussed below are therefore significant longer-term changes that occurred over a period of years.

The faunal assemblage seen throughout the time of deposition of Whipray core 25B is classified as a Brachidontes assemblage, typically what is found in Whipray Basin today (see Brewster-Wingard et al., in press, for an explanation of modern assemblages). No significant geographic shifts have occurred in the distribution of the molluscan assemblages within Florida Bay over the last 200 years. The shifts that have occurred have been changes in the dominance or presence-absence of given species within the same general assemblage. These subtle faunal changes, however, indicate important environmental changes within Whipray Basin and are the basis for the separation of the core into four zones. 
The molluscan assemblages generally indicate polyhaline to euhaline conditions throughout most of the core. Salinity did fluctuate frequently and appears to have been slightly lower in the bottom half of the core, where a few mesohaline species are present. Brachidontes exustus is a euryhaline species found alive in Florida Bay in salinities ranging from 10.2-41.3 ppt (Brewster-Wingard et al., in press). Field observations indicate Brachidontes is an opportunistic generalist that tolerates extreme fluctuations in salinity and diminished water quality. The persistence of this species throughout Whipray core $25 \mathrm{~B}$, and the near absence of species preferring clear, well-circulated water, such as Pteria longisquamosa, indicates the water of Whipray Basin has been restricted during the entire time of deposition of the core.

In zone 1, deposited between 1815-1857 $(78-60 \mathrm{~cm})$, faunal richness and abundance are relatively high. The majority of the species present are polyhaline (18-30 ppt), but periodic lower salinity conditions are indicated. Mesohaline species, Polymesoda maritima and Cerithidea costata, are present from 1815-1839 (78-68 cm), which suggest that salinity was lower during the early 1800s. In addition, the presence of Truncatella sp. and freshwater Hydrobiidae indicate proximity to an island or terrestrial influx.

Throughout zone 1, seagrass (Thalassia) and other sub-aquatic vegetation (macrobenthic algae and Halodule) were abundant, allowing epiphytic molluscs to flourish (Figure 4). Sub-aquatic vegetation was present and relatively abundant during the period of deposition for zone 1 (Figure 4). Epiphytic species account for $>70 \%$ of the molluscan fauna in this segment of the core. Bittiolum varium and Cerithium muscarum are present in higher percentages in this zone, compared to the overlying zones (Figure 3). Bittiolum varium is a sub-aquatic vegetation generalist, but is most typically found on mats of Polysiphonia, and also on Halodule, in very shallow areas $(<30 \mathrm{~cm})$. The high percentage of Bittiolum and the rare occurrence of Batillaria minima (also typically found in shallow areas with Halodule) in zone 1, indicate the water depth may have been shallower during deposition of this segment of the core, in contrast to the overlying zones.

Zone 2 represents a 32-year transitional period from 1862 to $1894(58-44 \mathrm{~cm})$. During this period, low-salinity (mesohaline) species are not present and the majority of species are polyhaline, suggesting a shift to more saline conditions. However, these conditions are not stable. The dominance of Brachidontes exustus indicates an increase in salinity fluctuations and/or diminished water quality. The period from 1862 to 1885 $(58-48 \mathrm{~cm})$ is characterized by a significant decrease in faunal richness and abundance (Figure 3); these measures of diversity reach a low point around $1883(50-48 \mathrm{~cm})$ due to a loss of the rare species present in zone 1.

The period around $1883(50-48 \mathrm{~cm})$ also corresponds to a significant drop in the sub-aquatic vegetation generalists and in the total epiphytic species (Figure 4). Several of the dominant species in the core undergo pronounced fluctuations (Figure 3), particularly Transennella. Our field investigations have yielded little data on Transennella, but it has 
Figure 4: Plot of down-core trends in molluscan epiphytic species separated into seagrass specialists, sub-aquatic vegetation generalists, and total epiphytes within core 25B.

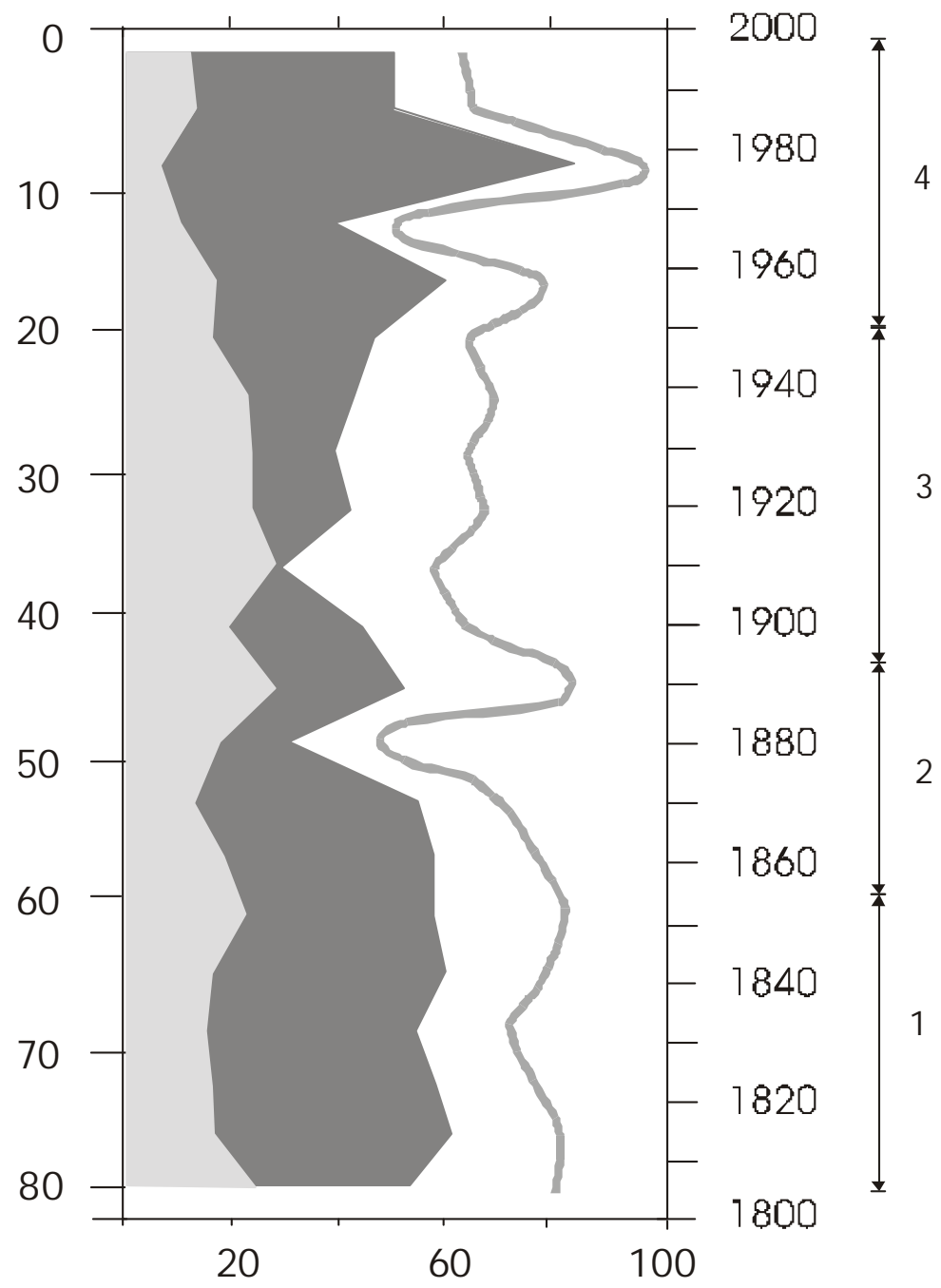

Seagrass specialists

SAV generalists

2 All SAV combined 
most commonly been found associated with "blowout areas." 4 A blowout could have caused the preceding drop in epiphytic species and allowed the increase of infaunal Transennella sp. The decline in sub-aquatic vegetation could also be a cause or an effect of diminished water quality (suggested due to an increase in Brachidontes). The end of the transitional period, from 1885 to $1894(48-44 \mathrm{~cm})$, is distinguished by a return of vegetation as indicated by an increase in epiphytic molluscs and in faunal richness and abundance.

Zone 3, from 1899 to $1950(42-20 \mathrm{~cm})$, is a period of relatively high diversity measures. Faunal richness and abundance values are high and constant, except for a peak in abundance around $1910(38-36 \mathrm{~cm})$. Faunal richness is high due to the presence of a group of epifaunal gastropods that are rare or absent in the rest of the core. Throughout this zone, many dominant species maintain steady populations (Figure 3). Salinity is also stable, with a polyhaline environment persisting during this time. The relatively low percent abundance of Brachidontes exustus supports the idea of stable salinities because Brachidontes exustus is believed to flourish under fluctuating conditions (based on unpublished field observations). This zone also corresponds to a period of low amplitude and low frequency fluctuations in the annual average rainfall for southern Florida ${ }^{5}$ (Figure 3). The presence of Marshallora nigrocincta indicates continuing proximity to an island.

Levels of sub-aquatic vegetation appear to fluctuate throughout this part of the core, based on the epiphytic species present (Figure 4). From 1894 to $1904(44-40 \mathrm{~cm})$, both seagrass and other sub-aquatic vegetation epiphytes experience a decline. By 1910 $(38-36 \mathrm{~cm})$, however, seagrass specialists reach their peak, while sub-aquatic vegetation generalists fall to their lowest percent abundance. By $1920(34-32 \mathrm{~cm})$, the abundance of sub-aquatic vegetation generalists increases and continues to rise until $1957(18-16 \mathrm{~cm})$. Seagrass specialists follow an inverse trend; their abundance slowly declines until 1976 $(10-8 \mathrm{~cm})$.

Zone 4, beginning in $1955(18 \mathrm{~cm})$, represents a period of profound change, unprecedented in lower portions of the core. A substantial decrease occurs in faunal richness and abundance. Many of the dominant species in the core (Figure 3) undergo extreme fluctuations. The fauna present generally indicate polyhaline conditions, but their rapid and high amplitude shifts in abundance imply a period of fluctuating salinities. Anomalocardia auberiana [=Anomalocardia cuniemeris Turney and Perkins, 1972] is a species typically found in the northern transition zone of Florida Bay (Brewster-Wingard et al., in press; Lyons 1996, 1999; Turney and Perkins, 1972). While it is characteristically found in lower salinities, it is believed to tolerate extreme fluctuations (based on unpublished field observations). The increase in Anomalocardia between 1964-1969 (14-12 cm), followed by the increase in Brachidontes exustus between 1974-

\footnotetext{
${ }^{4}$ Bare areas surrounded by dense grass beds with a very sharp transition zone are commonly referred to as "blowouts" in present-day Florida Bay. The cause of these "blowouts" is unknown, but some researchers have speculated it could be caused by seagrass die-off

${ }^{5}$ The rainfall data are based on a compilation of NOAA rainfall data from NOAA NCDC website http://www.ncdc.noaa.gov/., divisions 5, 6, and 7.
} 
$1978(10-8 \mathrm{~cm})$, provides strong evidence for a period of increased amplitude and frequency of salinity fluctuations. The rise in Acteocina canaliculata and Schwartziella catesbyana since $1974(10 \mathrm{~cm})$ is also significant, but field investigations have yielded little data on these species. The changes seen in this segment of the core may be related in part to rainfall (Figure 3). Beginning around 1947, the average annual rainfall data show increased amplitude and frequency of fluctuation in the amount of rain.

Molluscan epiphytes indicate sub-aquatic vegetation also fluctuates during the deposition of zone 4 . The abundance of sub-aquatic vegetation generalists drops between 1955 and 1969 (18-12 cm), but experiences a significant increase to reach its peak between 1974 and $1978(10-8 \mathrm{~cm})$ (Figure 4). At this time, seagrass specialists hit their lowest percent abundance, but do increase slightly at the top of the core. The pattern of decline, rebound, and decline in the sub-aquatic vegetation generalists is similar to the pattern seen between 1871 and $1913(54-36 \mathrm{~cm})$ in the core, but the pattern is far more pronounced in the later half on the $20^{\text {th }}$ century. The late 1980 's are a period of documented sea-grass die-off in central and western Florida Bay (Robblee, et al., 1991). The molluscan epiphytes, however, indicate that a long-term pattern of decline may have begun as early as 1910 if only the seagrass specialists are considered, and as early as 1955 for the total population of epiphytes (Figure 4).

\section{CONCLUSIONS}

Based on the molluscan assemblages from Whipray core 25B, Whipray Basin has had restricted water movement and polyhaline to euhaline (or hypersaline) salinities over the last 200 years, with slightly lower salinities during the early 1800's. Periods of increased salinity fluctuations have alternated with periods of increased stability. The period from 1815-1857 was generally polyhaline, possibly with brief periods of lower salinities, and relatively shallow water; molluscan faunal richness and abundance were stable. The transitional period from 1862 to 1894 was a period of instability, with a significant loss of molluscan biota and changes in the sub-aquatic vegetation. The most stable segment of the core is from 1899-1950; the molluscs were abundant and diverse, and the patterns of dominance did not change significantly during this period. From 1955 to the present, Whipray Basin has been characterized by fluctuating salinity, poor water quality (low O2, increased nutrients and/or reduced clarity) oxygen supply), and significant vegetation changes. Such changes may be related to increased fluctuations in average annual rainfall, which began around 1947.

The changes seen in the assemblages suggest that sub-aquatic vegetation has always been present at the core site. However, the decrease in seagrass specialists relative to the generalists since 1910 implies that Thalassia may have been declining while macro-benthic algae was increasing. From 1871-1913, molluscan epiphytic species go through a period of fluctuation, which indicates a significant change in the sub-aquatic vegetation. This pattern is repeated between 1955 and 1978, a period preceding the substantial die-off of Thalassia meadows in Rankin Lake just to the north of Whipray. These patterns raise the questions: 1) are the epiphytes responding to SAV changes; 2) is 
the shift from 1955 to 1978 related to the die-off? If the answer to both questions is yes, than the core preserves the record of a previous die-off that occurred in the late 1800's.

While the changes within Whipray Basin are significant, they have not been so profound that a complete turnover in faunal assemblages has occurred over the time period represented by the core. The dominant faunal components of the molluscan assemblage have remained the same; a Northern Transition Zone assemblage has not been replaced by an open bay assemblage. However, the dominance of individual species and the diversity patterns have changed over time indicating the environmental shifts within Whipray Basin. These findings are consistent with the patterns seen in other cores from Florida Bay (Brewster-Wingard, et al., in press), where the general assemblage type and the presence or absence of a given species has not changed significantly over the last two centuries, but the dominance of individuals within those assemblages have changed.

The causes of the changes in Whipray Basin may be natural, or they may be a combination of anthropogenic and natural factors. Whatever the cause, it is clear that the amount of change in the last 50 years exceeds the preceding 150 years. Restoration efforts need to focus on areas that have undergone profound changes in the later half of the $20^{\text {th }}$ century, and try to determine the causes and effects of those changes.

Paleoecologic studies, combined with geochemical and sedimentological analyses shed light on those patterns of change, and provide insight to the causes and effects. 


\section{REFERENCES CITED}

Abbott, R.T., 1974, American Seashells, 2nd Edition. Van Nostrand Reinhold Co. New York. 663 p.

Boyer, J.N., Fourqurean, J.W., and Jones, R.D., 1999, Seasonal and long-term trends in the water quality of Florida Bay (1989-1997): Estuaries, v. 22, n. 2B, p. 417-430.

Brewster-Wingard, G.L., Ishman, S.E., Willard, D.A., Edwards, L.E., and Holmes, C.W., 1997, Preliminary paleontologic report on cores 19A and 19B, from Russell Bank, Everglades National Park, Florida Bay: U.S. Geological Survey Open-file Report 97-460, 29 pp.

Brewster-Wingard, G.L., Ishman, S.E., Waibel, N.J., Willard, D.A., Edwards, L.E., and Holmes, C.W., 1998a, Preliminary paleontologic report on Core 37, from Pass Key, Everglades National Park, Florida Bay: U.S. Geological Survey Open-file Report 98-122, 22 pp.

Brewster-Wingard, G.L., Ishman, S.E., Holmes, C.W., 1998b, Environmental Impacts on the southern Florida Coastal Waters: A history of change in Florida Bay: Journal of Coastal Research, special issue 26, pp. 162-172.

Brewster-Wingard, G.L., and Ishman, S.E., 1999, Historical trends in salinity and substrate in central Florida Bay: A Paleoecological Reconstruction using modern analogue data: Estuaries, vol. 22, no. 2b, pp. 369-383.

Brewster-Wingard, G.L., Stone, J.R., and Holmes, C.W., in press, Molluscan faunal distribution in Florida Bay, past and present: an integration of down-core and modern data: Bulletin of American Paleontology, 38 ms.p.

Fourqurean, J.W., Zieman, J.C., and Powell, G.V.N., 1992, Phosphorous limitation of primary production in Florida Bay: evidence from $\mathrm{C}: \mathrm{N}: \mathrm{P}$ ratios of the dominant seagrass Thalassia testudinum: Limnology and Oceanography, v. 37, n. 1, p. 162171.

Holmes, C.W., Robbins, John, Halley, Robert, Bothner, Michael, Ten Brink, Marilyn, and Marot, Marci, in press, Sediment Dynamics of Florida Bay Mud Banks on a Decadal Time Scale: Bulletin of American Paleontology, 22 ms.p.

Ishman, S.E., Brewster-Wingard, G.L., Willard, D.A., Cronin, T.M., Edwards, L.E., and Holmes, C.W., 1996, Preliminary paleontologic report on Core T-24, Little Madeira Bay, Florida: U.S. Geological Survey Open-File Report 96-543, 27 pp.

Kovach, W.L., 1989, Comparisons of multivariate analytical techniques for use in prequaternary plant paleoecology: Review of Palaeobotany and Palynology, vol. 60, pp. 255-282. 
Kovach, W.L., 1995, Multivariate Data Analysis: in Statistical Modelling of Quaternary Science Data, D. Maddy and J.S. Brew, eds., Quaternary Research Association, Technical Guide, no. 5, pp. 1-38.

Lyons, W.G., 1996, An assessment of mollusks as indicators of environmental change in Florida Bay. Programs and Abstracts, Florida Bay Science Conference, December 1996, Key Largo, Florida, Florida Sea Grant, University of Florida, pp. $52-54$.

Lyons, W.G., 1999, Responses of benthic fauna to salinity shifts in Florida Bay: evidence from a more robust sample of the molluscan community: in Program and Abstracts, Florida Bay and Adjacent Marine Systems Science Conference, November 1999, Key Largo, Florida, p. 52-54.

McIvor, C.C., Ley, J.A., and Bjork, R.D., 1994, Changes in freshwater inflow from the Everglades to Florida Bay including effects on biota and biotic processes: a review in Ogden, J.C., and Davis, S.M., editors, Everglades: The ecosystem and its restoration: St. Lucie Press, Del Ray, FL, pp.117-146.

Perry, L.M., and Schwengel, J.S., 1955, Marine shells of the western coast of Florida: Ithaca, NY, Paleontological Research Institution, 318 p.

Robblee, M.B., Barber, T.R., Carlson, P.R., Jr., Durako, M.J., Fourqurean, J.W., Muehlstein, L.K., Porter, D., Yarbro, L.A., Zieman, R.T., and Zieman, J.T., 1991, Mass mortality of the tropical seagrass Thalassia testudinium in Florida Bay (USA): Marine Ecology Progress Series, v. 71, p. 297-299.

Turgeon, D.D., Quinn, J.F. Jr., Bogan, A.E., Coan, E.V., Hochberg, F.G., Lyons, W.G., Mikkelsen, P.M., Neves, R.J., Roper, C.F.E., Rosenberg, G., Roth, B., Scheltema, A., thompson, F.G., Vecchione, M., and Williams, J.D., 1998, Common and scientific names of aquatic invertebrates from the United States and Canada: mollusks, $2^{\text {nd }}$ edition. American Fisheries Society, Special Publication 26, Bethesda, MD, $526 \mathrm{p}$.

Turney, W.J. and Perkins, B.F., 1972, Molluscan distribution in Florida Bay. University of Miami, Miami, Florida, Sedimenta, vol. III, 37 pp.

Warmke, G.L., and Abbott, R.T., 1961, Caribbean Seashells: Narberth, PA, Livingston Publishing Co. 348 p.

Wingard, G.L., Ishman, S.E., Cronin, T.M., Edwards, L.E., Willard, D.A., and Halley, R.B., 1995, Preliminary analysis of down-core biotic assemblages: Bob Allen Keys, Everglades National Park, Florida Bay: U.S. Geological Survey Open-File Report 95-628, 35 pp. 